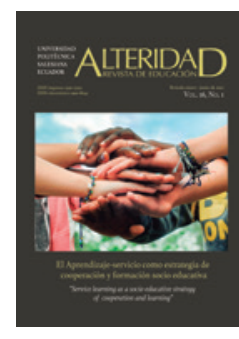

\title{
Metodología de aprendizaje-servicio en un proyecto integrado de costos y marketing
}

\author{
Service-learning methodology in an integrated cost and \\ marketing project
}

Dr. Francisco Ganga-Contreras es docente e investigador de la Universidad de Tarapacá (Chile) (franciscoganga@academicos.uta.cl) (https://orcid.org/0000-0001-9325-6459)

(D) Estela Rodríguez-Quezada es docente e investigadora de la Universidad de Bío-Bío (Chile) (erodrig@ubiobio.cl) (https://orcid.org/0000-0002-3259-0936)

(D) Nataly Guiñez-Cabrera es docente e investigadora de la Universidad de Bío-Bío (Chile) (nguinez@ubiobio.cl) (https://orcid.org/0000-0002-6109-8457)

Recibido: 2020-10-07 / Revisado: 2020-12-09 / Aceptado: 2020-12-14 / Publicado: 2021-01-01

\section{Resumen}

La formación de profesionales competentes, con visión y sello de compromiso con la sociedad, debe ir más allá de los procesos tradicionales de enseñanza, resultando entonces la innovación un factor clave. El aprendizaje-servicio (ApS) es una metodología que integra la enseñanza con el servicio comunitario de los estudiantes en un entorno real. Existen varios desafíos en la implementación de esta metodología activa, teniendo en cuenta las partes interesadas y las necesidades de la comunidad. En este sentido, este estudio busca describir la experiencia en cuanto al diseño, la implementación y la gestión de la metodología de aprendizaje-servicio en un proyecto integrado que contempla dos asignaturas, costos y marketing, en un programa de negocios en una universidad estatal del centro-sur de Chile. El trabajo se sustenta en la sistematización de experiencia, basada en tres fases de implementación dentro de la comunidad: (I) planificación, diseño y análisis, (2) entrega del servicio y, (3) evaluación, reflexión y monitoreo del aprendizajeservicio. Las etapas de diseño, implementación y gestión de un proyecto de estas características podrían servir de guía y orientaciones en futuras experiencias por parte de los profesores o las instituciones que se encuentren interesados en la innovación de los procesos de la enseñanza-aprendizaje.

Descriptores: Aprendizaje-servicio, costos, gestión educativa, gobernanza universitaria, implementación, marketing.

\section{Abstract}

The training of competent professionals, with a vision and stamp of commitment to society, must go beyond traditional teaching processes, with innovation being a key factor. Service-learning (S-L) is a methodology that integrates teaching with the community service of students in a real environment. There are several challenges in implementing this active methodology, taking into account the stakeholders and the needs of the community. In this sense, this study seeks to describe the experience regarding the design, implementation and management of the service-learning methodology in an integrated project that includes two subjects, costs and marketing, in a business program at a state university of the south-central Chile. The work is based on the systematization of experience, based on three phases of implementation within the community: (I) planning, design and analysis, (2) delivery of the service and, (3) evaluation, reflection and monitoring of service-learning. The stages of design, implementation and management of a project of these characteristics could serve as a guide and guidance in future experiences by teachers or institutions that are interested in the innovation of teaching-learning processes.

Keywords: Service-learning, costs, educational management, university governance, implementation, marketing.

Forma sugerida de citar: Ganga-Contreras, F., Rodríguez-Quezada, E., \& Guiñez-Cabrera, N. (2021). Metodología de aprendizaje-servicio en un proyecto integrado de costos y marketing. Alteridad, 16(1), 51-64. https://doi.org/10.17163/alt. v16n1.2021.04 


\section{Introducción}

Las organizaciones enfrentan un escenario caracterizado por profundas mutaciones sociales, políticas y económicas, fenómeno que se ha acrecentado dado el contexto de pandemia que está enfrentando el planeta, variables que generan inseguridades pues se ha incrementado la incertidumbre y obligado a la implantación de nuevos modelos (Ganga-Contreras, 2013; NiñoGonzález \& Linares-Herrera, 2020).

Es evidente que la generación, reproducción y transferencia de conocimientos, se mueve a velocidades impresionantes, en este mundo hipercomplejo y competitivo, vehiculizados por el profuso desarrollo de las tecnologías (GangaContreras et al., 2014; Ganga-Contreras et al., 2019a).

La realidad descrita, coloca el imperativo de ser cada vez más innovadores en la formación de los cuadros profesionales que necesita la comunidad donde se encuentra inserta la respectiva universidad con metodologías innovadoras y multifacéticas (Vidal-Raméntol \& FuertesCamacho, 2013; Sánchez-Marín et al., 2019) y aplicables a múltiples tipos de estudiantes (Puebla-Martínez et al., 2018) con propósitos educativos cada vez más amplios (Domínguez, 2018; Rubio, 2018).

El área de negocios no escapa a esta realidad y, por lo tanto, también se demandan profesionales que tengan una formación integral, con sensibilidad y empatía en el medio que se desenvuelven. En este contexto, nacen nuevas prácticas de hacer ciudadanía, formando a ciudadanos que sean participativos, responsables, y comprometidos con el bien común (Pérez-Galván \& OchoaCervantes, 2017).

Una metodología de enseñanza-aprendizaje que logra alcanzar este propósito es el aprendizaje-servicio (ApS), que intenta conectar de manera real al estudiante con la sociedad (Guiñez-Cabrera et al., 2020). Así, el ApS se ha logrado posicionar como una estrategia de educación profesional, que combina la formación académica y el servicio a la comunidad (GangaContreras et al., 2019b). Por lo tanto, formar ciudadanos críticos significa legitimar un proyecto educativo que se encuentre comprometido con la transformación social (Traver-Martí et al., 2019; Esteves-Fajardo et al., 2020). Como apunta Barrientos-Báez (2016), hoy se cuenta con unas herramientas que permiten que los procesos de aprendizaje sean mucho más personalizados y flexibles, como el mobile learning (AlisesCamacho, 2017) y el concepto de educomunicación (Rodríguez-García, 2017). En este caso concreto, la metodología ApS es un ejemplo del avance en estilos y herramientas de aprendizaje.

Existen varias definiciones de ApS, dependiendo del objetivo de estudio que se quiere lograr; puede entenderse como una experiencia educativa basada en cursos, donde los estudiantes son partícipes de actividades de un servicio organizado que satisfacen las necesidades identificadas de la comunidad (Orozco-Gómez et al., 2016).

La integración del servicio comunitario con el aprendizaje, enriquece los contenidos de los cursos y es evidente que los beneficios del ApS pueden tener un triple impacto positivo: estudiante, comunidad e institución (Al Barwani et al., 2013).

Las experiencias de ApS pueden ofrecer a los estudiantes de negocios una oportunidad para encontrar un sentido de responsabilidad personal, interactuar con la comunidad real, desarrollando mayores habilidades interpersonales, interculturales y sensibilidad ética (Simó-Algado et al., 2013; Carmona-Martínez et al., 2014) experimentando además los retos que regularmente demandan los proyectos reales, como la administración del tiempo, abordaje de las necesidades de los socios comunitarios y la colaboración con compañeros (Seider et al., 2011; Stefaniak, 2015). De este modo, Rodríguez-Izquierdo (2020) muestra que la metodología de enseñanza de ApS influye y mejora el compromiso académico de los estudiantes universitarios, mostrando actitudes más positivas hacia el estudio. 
Por otro lado, las instituciones se benefician del ApS - específicamente las escuelas de negocios- pueden generar contactos y contribuir a la comunidad, mejorando la imagen en el compromiso social, el vínculo con la comunidad y los logros por medio de los servicios comunitarios y la misión de educar a los estudiantes como personas socialmente responsables (Poon et al., 2011).

A su vez, la comunidad se puede beneficiar con esta pedagogía, pues las empresas tienen acceso a apoyos técnicos en materias específicas de administración.

Para este estudio en particular — se entenderá por ApS - aquella metodología pedagógica de enseñanza-aprendizaje basada en una o más asignaturas de un programa académico, que genera múltiples beneficios para todos los actores involucrados. Aunque existe un creciente interés en esta metodología, debido a sus grandes contribuciones, todavía no hay un marco de ejecución generalizado que pueda ser utilizado en cualquier contexto como en un proyecto integrado de dos asignaturas.

Considerando la falta de implementación de este tipo de proyectos en particular, este estudio tiene como propósito describir la experiencia del diseño, implementación y gestión de la metodología de ApS en un proyecto integrado de dos asignaturas (costos y marketing) en estudiantes de un programa de negocios en una universidad pública y estatal del centro-sur de Chile.

Para ello se recurre al modelo de ApS propuesto por Musa et al. (2017), el cual contempla tres fases: planificación, diseño y análisis del ApS; entrega del servicio de ApS; y; evaluación, reflexión y monitoreo del ApS.

Los resultados de este trabajo, podrían servir de guía para otras instituciones y profe- sores que quisieran implementar ApS y busquen mejorar sus procesos de enseñanza-aprendizaje a través de la innovación metodológica.

\section{Metodología de aprendizaje-servicio}

Dentro de los marcos de implementación, se encontró el trabajo de Pizarro et al. (2015) que muestran sus fases de implementación de la metodología de ApS, basándose en los postulados de Tapia (2007), donde la implementación de ApS fue en el curso de fundamento de costos realizado a partir de tres grandes fases: (1) búsqueda y clasificación de socios comunitarios, (2) implementación de la metodología y, (3) monitoreo y la evaluación. Así también, Petkus (2000), desarrolló un marco teórico-práctico para el ApS en el área de marketing, para guiar la planificación, la implementación y la evaluación de un curso de ApS en marketing por medio del modelo de ciclo de aprendizaje experimental de Kolb (1984).

Este proyecto integrado con ApS contempla dos asignaturas y se sustenta en el modelo de metodología de ApS propuesto por Musa et al. (2017), que se basa en tres fases de implementación: (1) planificación, diseño y análisis del ApS (implica identificar y analizar las necesidades y oportunidades para incorporarlas a los procesos y actividades de ApS), (2) entrega del servicio de ApS (enfocándose más en la implementación del proyecto integrado de ApS que tiene dos pasos, establecer la participación de la comunidad e institucionalizar el ApS dentro en la facultad, los estudiantes y la comunidad), (3) evaluación, reflexión y monitoreo del ApS (Ver Figura 1). 
Figura 1. Una metodología de implementación de ApS en un proyecto integrado

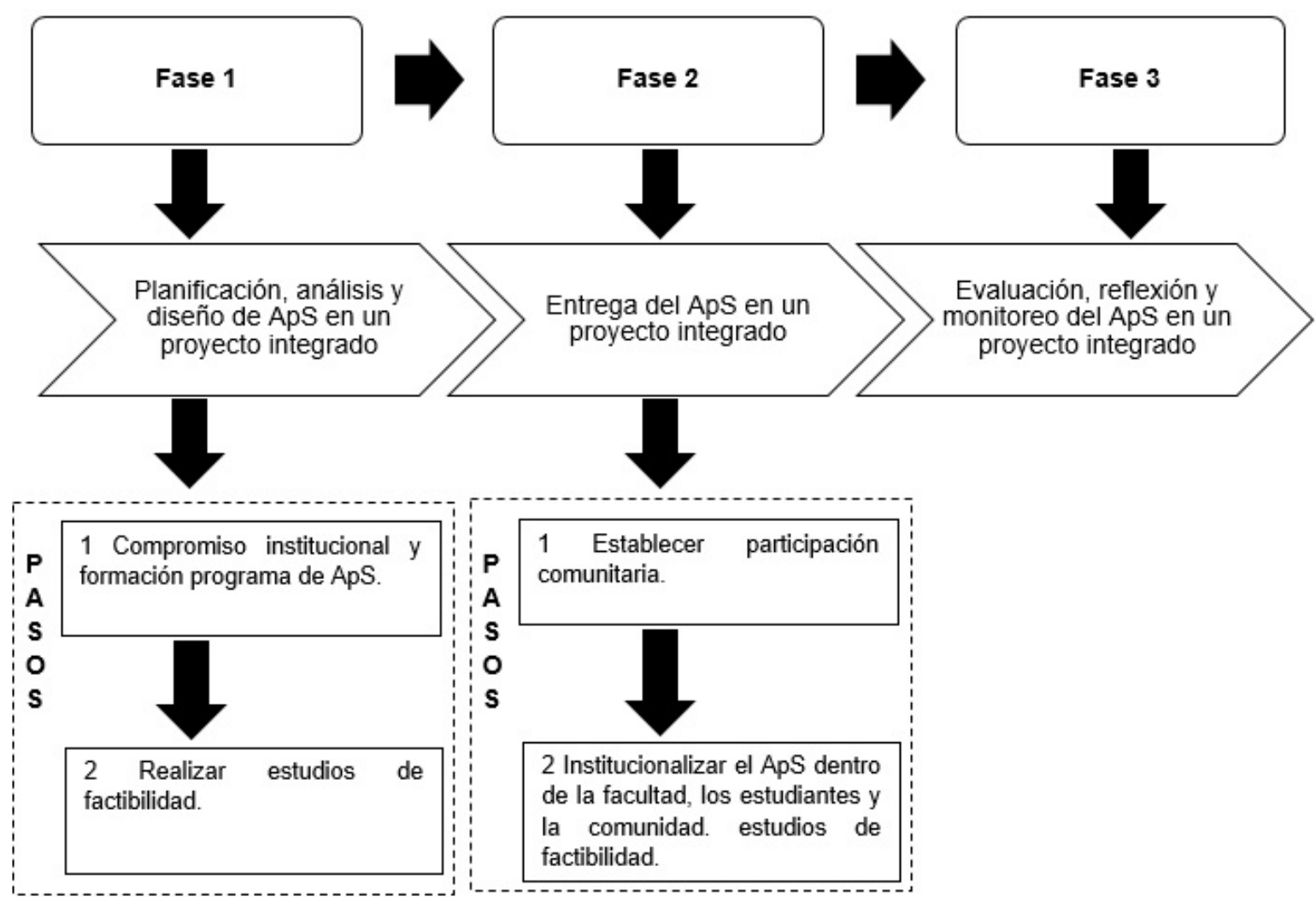

Fuente: Elaboración propia, basado en Musa et al. 2017.

\section{Propuesta}

\subsection{Fase 1: Planificación, análisis y diseño de ApS en un proyecto integrado}

Esta etapa, implica identificar y analizar las oportunidades y las necesidades para incorporarlas a los procesos y las actividades de ApS. Se distinguen dos pasos:

\subsubsection{Paso 1: Compromiso institucional y forma- ción programa de ApS}

El respaldo institucional es primordial para lograr buenos resultados en la implementación de la metodología de ApS (Guiñez-Cabrera et al., 2020). A su vez, es importante que los miembros de las instituciones comprendan que el ApS es un proceso efectivo para lograr objetivos académicos que son valorados por los estudiantes y la comunidad (Poon et al., 2011).

En el caso de la Universidad del Bío Bío (UBB), desde el 2008 posee un modelo educativo institucional, que centra la acción en el estudiante, preocupándose de su formación personal y profesional, buscando alcanzar un desarrollo integral como individuo, profesional y ciudadano, que le permite dar respuesta a las necesidades de su entorno. En este modelo, el proceso de enseñanzaaprendizaje, es fundamentalmente activo, donde la formación se orienta al logro de aprendizaje significativo. Un eje temático significativo es el compromiso, que corresponde a la responsabilidad profesional, personal y social, sobre la realidad económica y social; favoreciendo el desarrollo de la responsabilidad social por medio de la formación de profesionales que se encuen- 
tren comprometidos con su comunidad, con una mirada integradora de los problemas reales, velando por un desarrollo solidario. La formación y el reciclaje para un desarrollo profesional óptimo son piezas fundamentales para una organización (Barrientos-Báez et al., 2019; Ongallo-Chanción \& Gallego-Gil, 2020). Dentro de los componentes claves del aprendizaje, se considera el contexto experiencial, los conceptos procedimentales, teóricos y la puesta en práctica del conocimiento (Modelo Educativo UBB, 2017).

Por otro lado, Bringle y Hatcher (1996) sugieren que, durante la primera etapa de planificación, se necesita identificar la facultad interesada para desarrollar ApS e incluso formar un programa. El rol del programa de ApS de la facultad es hacer recomendaciones de los requisitos de ApS con respecto a los cursos, los estudiantes y la comunidad, siendo un vínculo entre la facultad y la comunidad (Musa et al., 2017).

La Facultad de Ciencias Empresariales (FACE) de la UBB, tiene un Plan de Mejoramiento denominado: "Desarrollo de competencias de los estudiantes de la Facultad de Ciencias Empresariales, a través de la metodología Aprendizaje Servicio y el uso de Tecnologías de la Información" (Programa Aprendizaje Servicio, 2017).

Como se sabe, las instituciones que se encuentran interesadas en el ApS, deben propiciar actividades de perfeccionamiento de los profesores, para desarrollar una comprensión común sobre el ApS y aumentar la confianza en la implementación de esta pedagogía (Bringle \& Hatcher, 1996). Sin el apoyo en la participación de los profesores, la incorporación del ApS en los planes de estudio no prosperará (Seifer \& Connors, 2007).

La UBB, posee un área de desarrollo pedagógico y tecnológico, y a su vez un programa permanente de pedagogía universitaria, a cargo de la unidad de gestión curricular y monitoreo donde proporciona capacitación a los profesores en variadas disciplinas, entre ellas se encuentra la metodología activa de ApS. Así también, el plan de mejoramiento de la FACE tiene como propósito fortalecer el aprendizaje y la gestión de los profesores basada en las experiencias reales (Programa Aprendizaje Servicio, 2017).

\subsubsection{Paso 2: Realizar estudios de factibilidad}

En el ApS, realizar un estudio de factibilidad es esencial antes de implementar el ApS en la comunidad (Musa et al., 2017). Hay cinco tipos de estudios de factibilidad que se deben llevar a cabo en la implementación del ApS; (1) Desarrollo del curso, (2) Proyectos de ApS, (3) Operativo, (4) Financiero, y (5) Técnico (Musa et al., 2017). Los estudios se describen a continuación.

\subsubsection{Desarrollo del curso}

El desarrollo de ApS dentro de la educación superior (ES) es principalmente un trabajo de los profesores (Bringle \& Hatcher, 1996). Los docentes necesitan estudiar cómo integrar el ApS dentro del plan de estudios en función de los requisitos y la dirección de las carreras. La preparación del curso es un proceso crucial en la implementación de ApS, revisando la remodelación del contenido del curso y los objetivos de aprendizaje donde los objetivos de ApS deben tener en cuenta los requisitos de los estudiantes y la comunidad (Musa et al., 2017).

El proyecto tiene que reflejar los contenidos del programa de estudios, las necesidades de la comunidad y la reflexión sobre las actividades de ApS (Gallgher et al., 1999). También, debe lograrse un acuerdo explícito y mutuo sobre los objetivos del ApS entre la facultad y la comunidad. La facultad también necesita estudiar los requisitos, las instrucciones, las actividades, los recursos de aprendizaje y las evaluaciones de los cursos, en este caso costos y marketing (Musa et al., 2017).

En primer lugar, las personas involucradas en el proyecto integrado, incorporaron las competencias del perfil genérico de la universidad como: disposición al aprendizaje y capacidad emprendedora (Mayer-Granados et al., 2019), 
liderazgo, trabajo colaborativo, capacidad para comunicarse y responsabilidad social; competencias que son fomentadas a través de la implementación de la metodología ApS. Y es que, según Barrientos-Báez et al. (2019), el nuevo modelo de enseñanzas aporta una manera diferente de entender la universidad y sus relaciones con la sociedad. La autonomía es la principal característica que las universidades tienen para responder con flexibilidad y rapidez a las cambiantes necesidades.

Luego, se revisaron las competencias del perfil de egreso, para garantizar que la implementación de esta metodología activa, tuviera concordancia con la formación del estudiante.

Después, se rediseñaron los respectivos cursos (costos y marketing), revisando los programas de las asignaturas para garantizar que el esquema del curso cumpliera con los contenidos abordados mediante ApS y las necesidades de los socios comunitarios. Adicionalmente, se analizó la metodología de ambas asignaturas, los contenidos actitudinales, conceptuales y procedimentales, y los criterios de evaluación; haciéndolos coherentes con las necesidades de los socios comunitarios. Los cursos fueron adecuados y relevantes para la comunidad, porque los objetivos de ApS enfatizan cómo los estudiantes de este programa de negocios, pueden proponer soluciones de costos y marketing para generar más oportunidades a las empresas de la zona, basándose en las necesidades reales de la sociedad donde están insertas.

También se consideró la respectiva carga académica (horas de trabajo) de los estudiantes; esta decisión permitió el alineamiento entre lo que especifica cada programa de las asignaturas (costos y marketing), con respecto a la carga semanal, que corresponde a cinco horas presenciales y cinco horas de trabajo autónomo semanales. Con respecto a la cantidad de tiempo en terreno, esta variable dependía de los requerimientos de los socios comunitarios, pero era requisito tener al menos una reunión presencial por semana, la cual dependía de la disponibili- dad del socio comunitario y el nivel de avance de los equipos de trabajo, siendo consideradas como parte del trabajo autónomo.

El proyecto integrado fue calificado con una nota en la escala de 1 a 7 y tenía una ponderación del 30\% de la calificación final en ambas asignaturas de costos y marketing. Una vez que se tuvo certeza de la efectividad de la implementación de la metodología, se continuó con la siguiente etapa.

\subsubsection{Proyectos de ApS}

Hay dos tipos de proyectos de ApS: servicios directos e indirectos (Gallgher et al., 1999). Este proyecto integrado fue un servicio directo que brindó apoyo a los socios comunitarios de manera presencial, satisfaciendo una necesidad determinada. Para lograrlo, los estudiantes debían comprender las necesidades que viven las empresas en la zona.

Esta implementación de ApS tenía la intención de mostrar a los estudiantes la relevancia del rol de los profesionales de negocios; se buscaba que ellos se convirtieran en verdaderos consultores para las empresas y otorgaran todos sus conocimientos de costos y marketing para mejorar los negocios asesorados.

Las asignaturas se diseñaron de tal manera que los estudiantes tendrían la oportunidad de lograr resultados como: comunicación efectiva, organización laboral, trabajo en equipo, identificación y solución de problemas, entre otros (Kearney, 2004).

La implementación de ApS en un curso de principios de marketing es probablemente el más desafiante, porque es muy probable que sea la primera exposición de los estudiantes a los conceptos y habilidades que se requieren en la comercialización; sin embargo, también es una excelente oportunidad para que estos alumnos principiantes experimenten la aplicación en el mundo real, transformándose en verdaderos consultores, evaluando los esfuerzos comerciales existentes e implementando planes estratégico 
donde se integran las diversas funciones de la mezcla de marketing (Petkus, 2000).

En lo referente a la asignatura de costos, los estudiantes podían realizar análisis de la documentación financiera identificando, clasificando, y calculando costos para los diferentes productos ofrecidos por las empresas; permitiendo poner en práctica conceptos teóricos relativos a la materia y vivenciar una experiencia real, además de complementar esto con el uso de las tecnologías de información y comunicación, al construir herramientas tecnológicas como las sugeridas por Pontes et al. (2020) que permitieron automatizar cada uno de los cálculos y crear diferentes escenarios de conveniencia para el servicio otorgado a los socios comunitarios.

\subsubsection{Operativo}

Se puede realizar un estudio de factibilidad operativa desde tres puntos de vista diferentes; facultad/ universidad, estudiante y comunidad (Musa et al., 2017). El estudio de viabilidad operativa debía considerar el compromiso institucional (Bringle \& Hatcher, 1996) y recursos. En este sentido, el plan de mejoramiento de la FACE, entregó apoyo a las profesoras en los cambios curriculares de ambas asignaturas y la orientación para la implementación de la metodología de ApS. Cuando se realizó la planificación, el equipo de las asignaturas, determinó el número de estudiantes por grupos, para poder lograr una correcta operatividad de la metodología de ApS; a su vez, desde la perspectiva de la comunidad, el estudio de factibilidad operativa identificó y determinó a los socios comunitarios más apropiados para la implementación de este proyecto integrado.

\subsubsection{Financiera}

El apoyo financiero requiere el compromiso de la institución (Seifer \& Connors, 2007). Un requisito financiero normalmente está determinado por el tamaño de las clases y la naturaleza del proyecto (Musa et al., 2017).
Gestionar catorce proyectos dentro de la comunidad fue un desafío y requiere una enorme cantidad de tiempo, trabajo y también recursos para lograr el objetivo final del ApS. La propuesta de presupuesto de ApS fue preparada en términos del diseño, planificación y costos involucrados, la cual se envió a la unidad responsable.

Después de presentar la propuesta de presupuesto de ApS, se recibió el respectivo soporte financiero de parte de la universidad, la Facultad de Ciencias Empresariales y el Departamento de Gestión Empresarial. La cantidad recibida por los patrocinadores se utilizó para el trabajo de campo, impresión de materiales, lanzamiento del proyecto integrado, trabajos administrativos $y$ relacionados; todo lo cual posibilitó la adecuada operacionalización de la metodología.

Tal como lo sugiere Musa et al. (2017), el plan de presupuesto se llevó a cabo garantizando que el proyecto integrado de ApS se pudiera implementar de manera efectiva y eficiente dentro de la comunidad.

El estudio de factibilidad financiera consideró los costos involucrados en la logística, donde se utilizaron las dependencias de la entidad educativa, específicamente el centro de extensión de la UBB, que se encuentra ubicado físicamente en un lugar céntrico y estratégico de la ciudad del centro-sur de Chile. El lanzamiento del proyecto y las presentaciones se realizaron en estas dependencias, para la facilidad y la comodidad, tanto de los empresarios (socios comunitarios) como de los estudiantes.

Dentro del trabajo administrativo, ambas profesoras de cada asignatura contaban con un ayudante respectivamente, para poder llevar a cabo de mejor manera el trabajo administrativo.

\subsubsection{Técnico}

El estudio de factibilidad técnica se relaciona con la cantidad de estudiantes que se pueden admitir para un buen desarrollo del proyecto de ApS (Musa et al., 2017), en este caso el proyecto integrado, contempló un total de 64 estudian- 
tes, resultando 14 equipos que trabajaron con el mismo número de empresas de la zona.

Dentro de la factibilidad técnica en el proyecto integrado, también se tuvo que identificar los requerimientos de tecnología y comunicación que demandaban los estudiantes, los socios comunitarios y los profesores. La UBB, realiza el préstamo de computadores portátiles a los estudiantes y profesores. El servicio de internet, los proyectores, la electricidad y el software utilizado para el análisis de datos se encontraban disponibles en la institución de educación.

\subsection{Fase 2: Entrega del ApS en un proyecto integrado}

Después de que todos los componentes en la fase 1 fueron considerados, la fase 2 se concentra en la implementación del proyecto integrado de ApS en las asignaturas, para ello se vale de dos pasos:

\subsubsection{Paso 1: Establecer participación comunitaria}

Crear una asociación entre la casa de estudios superiores y la comunidad es un proceso crucial para una participación comunitaria exitosa (Musa et al., 2017).

La participación comunitaria se describe como la colaboración entre la universidad y sus comunidades, para lograr el intercambio de recursos y conocimientos que sea mutuamente beneficioso en un contexto de asociación y reciprocidad (Driscoll, 2009).

Se necesita una asociación comunitaria bien planificada para garantizar que no solo estén dispuestas a participar, sino que también puedan colaborar eficazmente la comunidad y la respectiva entidad educativa. Durante el compromiso, tanto la comunidad como la universidad deben comprender y acordar mutuamente los beneficios de ApS para cada una de las partes (Musa et al., 2017).

Como la implicación preliminar y efectiva con los socios comunitarios es un proceso crucial, el equipo de ApS diseñó e implementó los siguientes pasos de participación de la comunidad y la institución de educación superior. En la primera reunión y lanzamiento del proyecto integrado de costos y marketing con ApS, se destacaron algunas preguntas, como: ¿qué es ApS?, ¿cómo el programa ApS beneficia a ambas partes?, ¿cuáles son los proyectos de costos y marketing que se podrían elaborar para la comunidad?, y ¿cuáles son las preferencias de proyecto que se relacionan con las necesidades?

En esta etapa, se utilizó un enfoque que lograra identificar las necesidades de los socios comunitarios. Como el ApS es una situación en la que todos ganan, los requisitos del contenido de los cursos, los conocimientos y las habilidades del estudiante se combinaron con la lista de proyectos de ApS propuestos por la comunidad. Ambos alcanzaron un acuerdo explícito y expectativas en términos de factibilidad de ejecutar por parte de los estudiantes a la comunidad.

\subsubsection{Paso 2: Institucionalizar el ApS dentro de la facultad, los estudiantes y la comunidad}

Después de identificar los requisitos de los cursos para trabajar con ApS, la macrounidad académica debe cultivar el ApS como parte de la cultura estudiantil (Musa et al., 2017). En esta línea de trabajo, la FACE, se adjudicó en el año 2014 un convenio de desempeño cuya finalidad es la ejecución y el desarrollo del Plan de Mejoramiento, financiado por el Banco Mundial a través del Ministerio de Educación de la República de Chile (Programa Aprendizaje Servicio, 2017). Dentro de sus propósitos se encuentra el perfeccionamiento de las competencias de los profesionales para la formación de los programas académicos de la Facultad con la metodología de ApS.

Otro objetivo fundamental apuntaba a mejorar la administración y el uso del equipamiento de TIC y de la infraestructura, para apoyar la gestión y el aprendizaje de los profesores a través de experiencias reales y con los contenidos de las respectivas asignaturas, implementando la metodología de ApS e instrumentos de eva- 
luación (Programa Aprendizaje Servicio, 2017). Es importante reseñar que las TIC ejercen una influencia cada vez mayor en los jóvenes ofreciendo la posibilidad de comunicarse interactivamente, de experimentar, de resolver problemas, de manejar todo tipo de datos y de simular situaciones reales (Barrientos-Báez, 2016).

La materialización de estas metodologías en las asignaturas fue a través de la incorporación de un proyecto integrado, que consistió en que los estudiantes debían asesorar a empresas pertenecientes a la Región de Nuble (Chile) en una necesidad concreta, conforme al perfil de egreso del estudiante de negocios, convirtiendo a los educandos en verdaderos consultores empresariales.

La evaluación de la actividad fue de manera sistemática y con todos los actores involucrados. Se esperaba que la incorporación de esta nueva metodología generara los siguientes resultados: mejoramiento del rendimiento académico en los estudiantes, aportes significativos en el desarrollo de habilidades sociales como trabajo colaborativo, comunicación efectiva oral y escrita (García-Roca, 2019), la búsqueda permanente de aprendizaje y establecimiento de redes de colaboración entre la universidad y las empresas del sector.

Para lograr este objetivo, se conformaron equipos de trabajo entre cinco y seis participantes con una población total de 64 estudiantes, resultando un total de 14 grupos de trabajo (dos secciones y ambas asignaturas).

La conformación de los equipos se realizó el primer día de clases utilizando para ello el cuestionario estilos de enseñanza-aprendizaje de VARK $^{1}$ y la metodología de aprendizaje basada en equipos (Team Based Learning), por lo cual se resguardó que la selección no fuese por afinidad y que los equipos de trabajo conformados fueran mixtos con distintos estilos de aprendizaje.

Los grupos de trabajo debieron definir un nombre para su equipo, y permanecieron permanente conformados durante todo el semestre y para todas las actividades que se desarrollaron en ambas asignaturas.
La asesoría del proyecto integrado de costos y marketing con ApS se desarrolló durante todo un semestre, los estudiantes tuvieron que contactar a una empresa con la cual decidirían trabajar en el proyecto y se planificó una reunión de trabajo mensual, entre los estudiantes, los empresarios y las profesoras en las dependencias de la universidad.

En la primera reunión de trabajo, se explicaron los objetivos del proyecto a los empresarios, definiendo los compromisos de ambas partes, tanto, de los estudiantes como de los empresarios para el exitoso desarrollo del proyecto integrado, y posteriormente cada equipo trabajó en conjunto para definir el problema a solucionar por parte de los estudiantes al interior de la empresa, también desarrollaron la planificación de las actividades (Carta Gantt) y la firma de acta de compromiso.

Por su parte los empresarios se comprometieron a facilitar toda la información que los estudiantes requirieran para el desarrollo del proyecto integrado, ejemplo: asistir mensualmente a las reuniones de trabajo programadas en la universidad y evaluar el desempeño de los grupos de trabajo; a su vez, los estudiantes y profesoras se comprometían a entregar un informe de asesoría que diera solución al problema planteado por el empresario en su empresa.

En cada reunión de trabajo los estudiantes presentaron un avance, de los aspectos que debía contemplar cada informe, de acuerdo con la planificación inicial, el que se entregaba en un informe escrito y luego se presentaba oralmente ante la comisión. La retroalimentación era inmediata en cada reunión de trabajo y posteriormente se calificaba tanto el informe escrito como la presentación.

\subsection{Fase 3: Evaluación, reflexión y monitoreo del ApS en un pro- yecto integrado}

Bringle y Hatcher (1996) —indican que los profesores y los estudiantes - pueden ser eva- 
luados utilizando el resultado del curso, como la satisfacción y el resultado del aprendizaje del estudiante, mientras que los socios comunitarios pueden evaluar el impacto de las actividades de ApS en sus necesidades satisfechas por los estudiantes en el proyecto integrado. El pensamiento de orden superior en cada etapa, es importante para enriquecer la experiencia de aprendizaje y potenciar la responsabilidad cívica y fortalecer a la comunidad (Seifer \& Connors, 2007).

Luego de las visitas a los socios comunitarios y el trabajo generado, los estudiantes regresaron a la Universidad para completar sus tareas y reflexionar en términos de las unidades de aprendizaje, desarrollo individual, habilidades blandas de comunicación y habilidades técnicas. Además, revisaban su bitácora de guía por cada visita, completaban un formulario de reflexión y un libro de registro. En la reflexión se obtuvo información de la experiencia vivida, el conocimiento y grado de comprensión de los cursos. las habilidades adquiridas y el impacto en la comunidad del proyecto integrado con ApS.

Se realizaron un total de cuatro presentaciones con entrega de informes escritos con los avances. Los aspectos evaluados pueden ser observados en la figura 2.

Figura 2. Aspectos evaluados en la aplicación de Aps

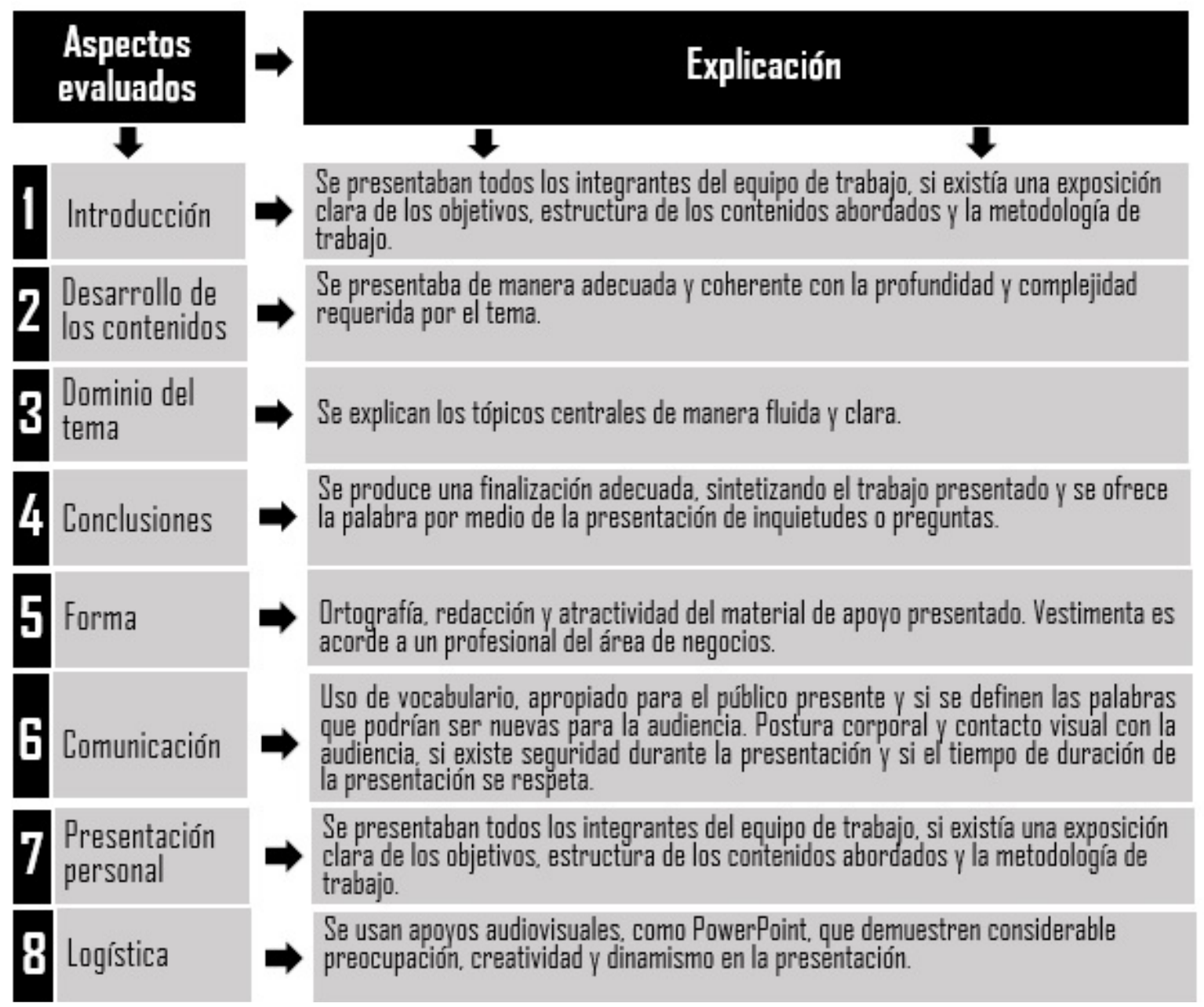

Fuente: Elaboración propia, basado en implementación de ApS. 
Para evaluar el impacto de esta metodología de ApS se utilizaron técnicas de investigación cualitativa-cuantitativa. El instrumento usado fue una encuesta de autoeficacia aplicada a los estudiantes al final del proyecto integrado.

Para hacer una reflexión, en términos de impacto comunitario y las relaciones universidad-comunidad (Musa et al., 2017), a los empresarios se les aplicó una encuesta de satisfacción al cierre del proyecto que constaba de once preguntas de las cuales seis eran cerradas y cinco preguntas abiertas que buscaban obtener su opinión respecto a la experiencia vivida con el proyecto, calificando la experiencia vivida de trabajar en este proyecto y evaluando las habilidades observadas en los estudiantes.

\section{Conclusiones}

Está claro que la metodología de ApS en un programa de negocios, mediante un proyecto integrado, tiene múltiples beneficios para todos los actores involucrados. Los estudiantes, al poder aplicar de manera práctica los contenidos entregados teóricamente en el aula, enriquecen su aprendizaje al adquirir experiencias desde el mundo real. Los socios comunitarios pueden abordar necesidades no satisfechas y las instituciones pueden generar acciones concretas de vinculación con el medio.

Sin embargo, además de conocer los beneficios de esta metodología innovadora, se debe considerar que dentro de la implementación de este proceso existen muchas aristas que son relevantes conocer y considerar para una buena ejecución de esta metodología de ApS.

Este estudio describió un proceso completo y detallado sobre el diseño, la implementación y la gestión de una metodología de ApS que contempló dos asignaturas, (costos y marketing) de la carrera de Ingeniería Comercial de la FACE de la UBB.

En este trabajo se presentaron pormenorizadamente cada una de las fases aplicadas durante el proceso con sus respectivas sub-etapas, todas las cuales son fundamentales para poder lograr un buen diseño, implementación y gestión de la metodología ApS en un proyecto integrado. Ninguno de los pasos es más importante que otros, por lo mismo, se estima que todos son vitales de llevarlos a cabo para poder lograr el objetivo deseado de tener una contribución a la enseñanza-aprendizaje significativa por medio de esta pedagogía innovadora.

Al realizar comparaciones con otros estudios se encuentra, por ejemplo, que Pizarro et al. (2015) indican que la implementación de ApS se basa en tres fases (búsqueda y clasificación de socios comunitarios, implementación de la metodología, y monitoreo-evaluación); del mismo modo, Petkus (2000) desarrolló un marco teórico-práctico para guiar un curso en marketing con ApS. Al analizarlos en detalles, se pueden encontrar varias diferencias con los modelos indicados anteriormente:

Se aplica esta metodología en una sola asignatura (en este estudio son dos).

El trabajo de Pizarro et al. (2015) indica tres fases, donde la primera corresponde a la búsqueda de los socios comunitarios, luego viene la fase de implementación de la metodología donde se vincula al socio comunitario con los estudiantes, y se entrega el producto de asesoría y finalmente viene el monitoreo y evaluación. La diferencia con la propuesta presentada se encuentra en las fases y especificación de cada una de ellas, donde este artículo va una etapa anterior que corresponde al compromiso institucional y los estudios de factibilidad.

Por su parte, el trabajo de Petkus, tiene como objetivo proporcionar un marco para la diseño e implementación de cursos de ApS en marketing, pero principalmente basándose en el programa de la asignatura en sí, no incorporando otras variables más bien operativas que son indispensables en el proceso de una buena implementación.

En vista de los antecedentes, se puede destacar que el gran aporte de este estudio, está en detallar cada fase, que comienza con una fase de 
factibilidad, dado que la mayoría de los modelos que se plantean para incorporar esta metodología de ApS comienzan desde la etapa de buscar socios comunitarios o de verificar si la metodología se ajusta con el programa de la asignatura. Este estudio hace hincapié en que esas fases son importantes, pero también, es necesario llevar a cabo estudios previos de factibilidad para conocer si será posible lograr los objetivos de una o varias asignaturas que incorporen esta metodología innovadora.

Se espera que los hallazgos de este estudio sean útiles para avanzar en nuestra comprensión de cómo diseñar, implementar y gestionar proyectos integrados con ApS, otorgando de este modo valor a todos los actores involucrados y al alcance de los objetivos de enseñanza-aprendizaje.

Si bien este estudio proporciona gran utilidad al describir la experiencia de una metodología innovadora en un proyecto integrado, también presenta una serie de limitaciones. Una de ellas apunta al diseño, la implementación y la gestión de la metodología de ApS en dos asignaturas concretas del área de negocios y en un solo contexto que es el chileno.

Por otro lado, la implementación de esta metodología se llevó a cabo en una institución de educación superior que cuenta con el apoyo a este tipo de metodologías, y que incluso tiene un programa institucional de ApS. Por lo tanto, en otro tipo de institución las fases para llevar a cabo este tipo de metodología podrían ser distintas o muy difíciles de operacionalizar.

Investigaciones futuras podrían realizarse en asignaturas de otras áreas, en otros contextos de Latinoamérica y en otro tipo de instituciones, para verificar si las fases identificadas en este estudio se cumplen o tienen diferencias significativas.

\section{Nota}

1. Visual, Aural, Read/Write, Kinesthetic por sus siglas en inglés.

\section{Referencias bibliográficas}

Al-Barwani, T., Al-Mekhlafi, A., \& Nagaratnam, R.P. (2013). Service-Learning Might be the Key: Learning from the challenges and implementation strategies in EFL Teacher Education in Oman. International Journal of Instruction, 6(2), 109-128. http://bit.ly/barwanietal

Alises-Camacho, M.E. (2017). Potencial pedagógico del Mobile Learning en el aula de música en secundaria. Revista de Comunicación de la SEECI, 43, 29-51. http://dx.doi.org/10.15198/seeci.2017.43.29-51

Barrientos-Báez, A. (2016). GDS Amadeus. Propuesta de innovación didáctica. En TIC actualizadas para una nueva docencia universitaria. McGraw Hill.

Barrientos-Báez, A., Barquero-Cabrero, M., \& Rodríguez-Terceño, J. (2019). La educación emocional como contenido transversal para una nueva política educativa: el caso del grado de turismo. Revista Utopía y Praxis Latinoamericana, 24(4), 147-165. https://bit. ly/3gMTolG

Bringle, R.G., \& Hatcher, J.A. (1996). Implementing service learning in higher education. Journal of Higher Education, 67(2), 221-239. https:// doi.org/10.1080/00221546.1996.11780257

Carmona-Martínez, M., Conesa-Pérez, M. del C., \& Ros-Clemente, M.I. (2014). Valoración del Aprendizaje basado en Problemas por los alumnos: diferencias por sexo. Historia $y$ Comunicación Social, 19, 725-734.

https://doi.org/10.5209/rev_HICS.2014.v19.44997

Domínguez, A. (2018). Fomento de la lectura y la escritura en lenguas indígenas de México: algunas consideraciones. Investigaciones Sobre Lectura, 10, 55-94. https://bit.ly/387X6ST

Driscoll, A. (2009). Carnegie's new community engagement classification: Affirming higher education's role in community. New Directions for Higher Education, 5-12. https:// doi.org/10.1002/he.353

Esteves-Fajardo, Z., Chenet-Zuta, M.E., PibaquePonce, M.S., \& Chávez-Rocha, M.L. (2020). Estilos de aprendizaje para la superdotación en el talento humano de estudiantes universitarios. Revista de Ciencias Sociales, XXVI(2), 225-235. https://bit.ly/34ffqbN 
Ganga-Contreras, F. (2013). Gerencia latinoamericana en las actuales plataformas organizacionales: Una mirada preliminar y sinóptica. Revista Venezolana de Gerencia, 18(64), 711722. https://bit.ly/Ganga-Contreras

Ganga-Contreras, F., Piñones, M. \& Valderrama, C. (2014). Innovaciones teóricas en administración: una sinóptica mirada diacrónica. Prisma Social, 12, 688-707.

https://bit.ly/Ganga-Contrerasetal

Ganga-Contreras, F., Rodríguez-Ponce, E., PedrajaRejas, L., \& Alarcón, N. (2019a). Percepciones de los funcionarios públicos sobre la relación entre Universidades Públicas y Privadas con el Estado De Chile. Interciencia, 44(9), 521 528. https://bit.ly/Ganga-Contrerasetal2

Ganga-Contreras, F., Guiñez-Cabrera, N., OlguínGutiérrez, C., \& Ceballos-Garrido, P. (2019b). Percepción estudiantil de la metodología "aprendizaje-servicio" en la asignatura de marketing. Opción, 35(90), 475-505. http://bit.ly/gangacontrerasetal

García-Roca, A. (2019). Los fanfictions como escritura en colaboración: modelos de lectores beta. El profesional de la información, 28(4). https://doi.org/10.3145/epi.2019.jul.04

Guiñez-Cabrera, N., Ganga-Contreras, F., OlguínGutiérrez, C., \& Ceballos-Garrido, P. (2020). Metodología de Aprendizaje Servicio: Experiencia de implementación desde la perspectiva de marketing. Revista Academia y Negocios, 6(1), 1-10.

http://bit.ly/guinezcabreraetal

Kearney, K.R. (2004). 'Students' Self-Assessment of Learning through Service-Learning'. American Journal of Pharmaceutical Education, 68(1), 1-13. http://bit.ly/kearneykr

Kolb, D.A. (1984). Experiential learning: Experience as the source of learning and development. Prentice Hall. http://bit.ly/kolb1984

Mayer-Granados, E.L., Charles-Coll, J.A., \& De la Garza-Ramos, M.A. (2019). El fomento emprendedor desde la universidad mexicana. Revista Venezolana de Gerencia, 24(85), 49-67. https://bit.ly/380eb0U

Modelo Educativo de la Universidad del Bío Bío. https://bit.ly/ModeloEducativoUBB

Musa, N., Ibrahim, D.H.A., Abdullah, J., Saee, S., Ramli, F., Mat, A.R., \& Khiri, M.J.A. (2017). A methodology for implementation of service learning in higher education institution: A case study from faculty of computer science and information technology, UNIMAS. Journal of Telecommunication, Electronic and Computer Engineering, 9(2-10), 101-109. http://bit.ly/musaetal

Niño-González, J.I., \& Linares-Herrera, M.P. (2020). Investigar: Acción impostergable. Bibliotecas. Anales de Investigación, 16(1), 5-6. https://bit.ly/384PUqJ

Orozco-Gómez, M., Sánchez-Fuentes, S., CuestaGómez, J.L., Cifuentes-García, A., \& MartínAlmaraz, R.A. (2016). Participación en comunidades de aprendizaje, como elemento clave para la formación inicial en el ámbito de la educación inclusiva e intercultural. Revista de Ciencias de la Comunicación e Información, 21(1), 35-44. http://doi.org/10.35742/rcci.2016.21(1).35-44

Ongallo-Chanción, C., \& Gallego-Gil, D. (2020). El 'emofeedback': la inteligencia emocional y el feedback en los procesos de acompañamiento. Revista de Ciencias de la Comunicación e Información, 25(2), 1-22. https://doi.org/10.35742/rcci.2020.25(2).1-22

Pérez-Galván, L.M., \& Ochoa-Cervantes, A. de la C. (2017). El aprendizaje-servicio (APS) como estrategia para educar en ciudadanía. Alteridad, 12(2). https://doi.org/10.17163/alt.v12n2.2017.04

Petkus, E. (2000). A Theoretical and Practical Framework for Service-Learning in Marketing: Kolb's Experiential Learning Cycle. Journal of Marketing Education, 22(1), 64-70. https://doi.org/10.1177/0273475300221008

Pizarro-Torres, V., Hasbun-Held, B., \& GonzálezCifuentes, T. (2015). Innovación en docencia: metodología de "aprendizaje y servicio" en contabilidad de costos. CAPIC REVIEW, 13, 89-99. https://doi.org/10.35928/cr.vol13.2015.47

Poon, P., Chan, T.S., \&Zhou,L.(2011). Implementation of Service-Learning in Business Education: Issues and Challenges. Journal of Teaching in International Business, 22(3), 185-192. https://doi.org/10.1080/08975930.2011.653746

Pontes, N., Dorado-Santana, \& Baptista-Calunga, A. (2020). A gestão documental em instituições escolares: directrizes para o desenho de um 
sistema de gestão electrónica de documentos escolares. Bibliotecas. Anales de Investigación, 16(1), 23-45. https://bit.ly/3nmtZln

Programa Aprendizaje Servicio de la Facultad de Ciencias Empresariales, Universidad del Bío Bío. https://bit.ly/ApSFACE

Puebla-Martínez, B., Rodas-Alfaya, L., \& Guede-Cid, R. (2018). Principales factores que intervienen en el diseño de los planes de aprendizaje en materia digital para mayores de 65 años. Revista Opción, Especial(18), 1929-1956. https://bit.ly/3a9nQFk

Rodríguez-Izquierdo, R.M. (2020). Aprendizaje Servicio y compromiso académico en Educación Superior. Revista de Psicodidáctica, 25(1), 45-51.

https://doi.org/10.1016/j.psicod.2019.09.001

Rodríguez-García, Y.M. (2017). Reconceptualización de la educación en la era digital: Educomunicación, redes de aprendizaje y cerebro factores claves en los actuales escenarios de construcción de conocimiento. Revista de Comunicación de la SEECI, 42, 85-118. https://doi.org/10.15198/seeci.2017.42.85-118

Rubio, A. (2018). Técnicas de aprendizaje de conocimiento científico a partir de textos. Investigaciones sobre Lectura, 10, 1-29. https://bit.ly/38mH7AL

Sánchez-Marín, F.J., Parra-Meroño, M.C., \& PeñaAcuña, B. (2019). Experiencias de trabajo cooperativo en la educación superior. Percepciones sobre su contribución al desarrollo de la competencia social. Vivat Academia, Revista de Comunicación, 147, 87-108. http://doi.org/10.15178/va.2019.147.87-108.

Seider, S.C., Gillmor, S.C., \& Rabinowicz, S.A. (2011). The Impact of Community Service Learning
Upon the Worldviews of Business Majors Versus Non-Business Majors at an American University. Journal of Business Ethics, 98, 485-503. https://doi.org/10.1007/s10551-010-0589-8

Seifer, S.D., \& Connors, K. (2007). Faculty Toolkit for Service-Learning in Higher Education. National Service Learning Clearinghouse. http://bit.ly/seiferandconnors

Simó-Algado, S., Ginesta-Portet, X., \& de San Eugenio-Vela, J. (2014). Aprendizaje Servicio Universitario: Creando empleo a partir de la emprendeduría social. Historia y Comunicación Social, 18, 627-638. https://doi.org/10.5209/rev_HICS.2013.v18.44265

Stefaniak, J.E. (2015). The implementation of servicelearning in graduate instructional design coursework. Journal of Computing in Higher Education, 27, 2-9. https://doi.org/10.1007/s12528-015-9092-7

Tapia, M.N. (2007). El aprendizaje-servicio en las organizaciones de la sociedad civil. En Programa Nacional Educación Solidaria. Aprendizaje y Servicio Solidario en las organizaciones de la sociedad civil. Programa Nacional Educación Solidaria. https://bit.ly/ApSenlasorgs

Traver-Martí, J.A., Moliner-García, O., \& SalesCiges, A. (2019). Negociando el currículum: Aprendizaje-servicio en la escuela incluida. Alteridad, 14(2), 195-206. https://doi. org/10.17163/alt.v14n2.2019.04

Vidal-Raméntol, S., \& Fuertes-Camacho, M.T. (2013). La dinámica de grupos para el trabajo cooperativo facilita la comunicación. Revista de Comunicación VIVAT Academia, 123, 1-12. https://doi.org/10.15178/va.2013.123.1-12 